Research Article

\title{
Generalization of Some Simpson-Like Type Inequalities via Differentiable s-Convex Mappings in the Second Sense
}

\author{
Jaekeun Park \\ Department of Mathematics, Hanseo University, Seosan-Si, Chungnam-Do 356-706, Republic of Korea \\ Correspondence should be addressed to Jaekeun Park, jkpark@hanseo.ac.kr
}

Received 31 March 2011; Accepted 24 June 2011

Academic Editor: Jewgeni Dshalalow

Copyright (C) 2011 Jaekeun Park. This is an open access article distributed under the Creative Commons Attribution License, which permits unrestricted use, distribution, and reproduction in any medium, provided the original work is properly cited.

The author obtained new generalizations and refinements of some inequalities based on differentiable s-convex mappings in the second sense. Also, some applications to special means of real numbers are given.

\section{Introduction}

Recall that the function $f:[0, b] \rightarrow \mathbb{R}, b>0$ is said to be s-convex in the second sense for $s \in[0,1]$ if

$$
f(t x+(1-t) y) \leq t^{s} f(x)+(1-t)^{s} f(y)
$$

for all $x, y \in[0, b]$ and $t \in[0,1][1-5]$.

In (1.1), if we let $s=1, f: \mathbb{I} \subset[0, \infty) \rightarrow \mathbb{R}$ is said to be a convex mapping on an interval $\mathbb{I}[1]$.

Let us denote the set of s-convex mappings in the second sense on $\mathbb{I}$ by $K_{S}^{2}(\mathbb{I})$.

For some further properties of the s-convex mappings, see $[1-3,6]$. In recent, M. Z. Sarikaya et al. [4], and U. S. Kirmaci et al. [7] established a more general result of the HermiteHadamard inequalities.

For recent years many authors have established error estimations for the Simpson's inequality: for refinements, counterparts, generalizations, and new Simpson's type inequalities, see $[1,4,6,8]$. 
S. S. Dragomir et al. [9], and M. Alomari et al. [8] proved the following developments on Simpson's inequality for which the remainder is expressed in terms of lower derivatives than the twice.

In the sequel, denote the interior of an interval $\mathbb{I}$ by $\mathbb{I}^{0}$.

Theorem 1.1. Let $f: \mathbb{I} \subset[0, \infty) \rightarrow \mathbb{R}$ be an absolutely continuous mapping on $[a, b]$ such that $f^{\prime} \in L_{p}([a, b])$, where $a, b \in \mathbb{I}$ with $a<b$. Then the following inequality holds:

$$
\left|\frac{1}{3}\left\{\frac{f(a)+f(b)}{2}+2 f\left(\frac{a+b}{2}\right)\right\}-\frac{1}{b-a} \int_{a}^{b} f(x) d x\right| \leq \frac{(b-a)^{1 / q}}{6}\left\{\frac{2^{q+1}+1}{3(q+1)}\right\}^{1 / q}\left\|f^{\prime}\right\|_{p} .
$$

In this article, the author gives some generalized Simpson's type inequalities based on $s$-convex mappings in the second sense by using the following lemma.

\section{Generalization of Inequalities Based on s-Convex Mappings}

In this article, for the simplicity of the notation, let

$$
S_{a}^{b}(f)(h, n)=\frac{1}{n}\{f(a)+(n-2) f(h b+(1-h) a)+f(b)\}-\frac{1}{b-a} \int_{a}^{b} f(x) d x,
$$

for $h \in(0,1)$ with $1 / n \leq h \leq(n-1) / n$ for any integer $n \geq 2$.

In order to generalize the classical Simpson-like type inequalities, we need the following lemma $[1,6]$.

Lemma 2.1. Let $f:[0, b] \rightarrow \mathbb{R}, b>0$ be a differentiable mapping on $\mathbb{I}^{0}$ such that $f^{\prime} \in L[a, b]$, where $a, b \in \mathbb{I}$ with $a<b$ and $[a, b] \subset[0, b]$. If $f^{\prime} \in L^{1}([a, b])$, then, for $h \in(0,1)$ with $1 / n \leq h \leq$ $(n-1) / n$ for any $n \geq 2$ the following equality holds:

$$
S_{a}^{b}(f)(h, n)=(b-a) \int_{0}^{1} p(t, h) f^{\prime}(t b+(1-t) a) d t
$$

for each $t \in[0,1]$, where

$$
p(t, h)= \begin{cases}t-\frac{1}{n}, & t \in[0, h] \\ t-\frac{n-1}{n}, & t \in(h, 1]\end{cases}
$$


Proof. By the integration by parts, we have

$$
\begin{aligned}
\int_{0}^{h}\left(t-\frac{1}{n}\right) f^{\prime}(t b+(1-t) a) d t+\int_{h}^{1}\left(t-\frac{n-1}{n}\right) f^{\prime}(t b+(1-t) a) d t \\
=\frac{1}{b-a}\left\{\frac{n-2}{n} f(h b+(1-h) a)+\frac{1}{n}(f(a)+f(b))\right\}-\frac{1}{(b-a)^{2}} \int_{a}^{b} f(x) d x
\end{aligned}
$$

which completes the proof.

Theorem 2.2. Let $f:[0, b] \rightarrow \mathbb{R}, b>0$ be a differentiable mapping on $\mathbb{I}^{0}$ such that $f^{\prime} \in L[a, b]$, where $a, b \in \mathbb{I}$ with $a<b$ and $[a, b] \subset[0, b]$. If $\left|f^{\prime}\right| \in K_{s}^{2}([a, b])$, for some $s \in(0,1]$, then for $h \in(0,1)$ with $1 / n \leq h \leq n-1 / n$ for any $n \geq 2$ the following inequality holds:

$$
\left|S_{a}^{b}(f)(h, n)\right| \leq(b-a)\left\{\lambda_{11}\left|f^{\prime}(b)\right|+\mu_{11}\left|f^{\prime}(a)\right|\right\},
$$

where

$$
\begin{aligned}
\lambda_{11}= & \frac{2+2(n-1)^{s+2}}{n^{s+2}(s+1)(s+2)}+\frac{2+s-n}{n(s+1)(s+2)} \\
& +\frac{h^{s+1}(s(2 h-1)+2(h-1))}{(s+1)(s+2)}, \\
\mu_{11}= & \frac{2+2(n-1)^{s+2}}{n^{s+2}(s+1)(s+2)}+\frac{2+s-n}{n(s+1)(s+2)} \\
& +\frac{(1-h)^{s+1}\{s(1-2 h)-2 h\}}{(s+1)(s+2)} .
\end{aligned}
$$

Proof. From Lemma 2.1 and since $\left|f^{\prime}\right|$ is s-convex on $[a, b]$, by using Hölder integral inequality, we have

$$
\begin{aligned}
\left|S_{a}^{b}(f)(h, n)\right| \leq & (b-a) \int_{0}^{1 / n}\left(\frac{1}{n}-t\right) \mid\left(t^{s}\left|f^{\prime}(b)\right|+(1-t)^{s}\left|f^{\prime}(a)\right|\right) d t \\
& +(b-a) \int_{1 / n}^{h}\left(t-\frac{1}{n}\right)\left(t^{s}\left|f^{\prime}(b)\right|+(1-t)^{s}\left|f^{\prime}(a)\right|\right) d t \\
& +(b-a) \int_{h}^{(n-1) / n}\left(\frac{n-1}{n}-t\right)\left(t^{s}\left|f^{\prime}(b)\right|+(1-t)^{s}\left|f^{\prime}(a)\right|\right) d t \\
& +(b-a) \int_{(n-1) / n}^{1}\left(t-\frac{n-1}{n}\right)\left(t^{s}\left|f^{\prime}(b)\right|+(1-t)^{s}\left|f^{\prime}(a)\right|\right) d t \\
= & (b-a)\left\{\lambda_{11}\left|f^{\prime}(b)\right|+\mu_{11}\left|f^{\prime}(a)\right|\right\}
\end{aligned}
$$

which implies the theorem. 
Corollary 2.3. In Theorem 2.2, one has:

(i)

$$
\left|S_{a}^{b}(f)\left(\frac{1}{2}, n\right)\right| \leq(b-a)\left\{\frac{2+2(n-1)^{s+2}+n^{s+1}\left(s-n+2-2^{-(s+1)} n\right)}{n^{s+2}(s+1)(s+2)}\right\}\left\{\left|f^{\prime}(a)\right|+\left|f^{\prime}(b)\right|\right\},
$$

(ii)

$$
\left|S_{a}^{b}(f)\left(\frac{1}{2}, 6\right)\right| \leq(b-a)\left\{\frac{6^{-s}\left(1+5^{s+2}-3^{s+2}\right)+3(s-4)}{18(s+1)(s+2)}\right\}\left\{\left|f^{\prime}(a)\right|+\left|f^{\prime}(b)\right|\right\},
$$

which implies that Corollary 2.3 is a generalization of Theorem 1.1.

Theorem 2.4. Let $f:[0, b] \rightarrow \mathbb{R}, b>0$ be a differentiable mapping on $\mathbb{I}^{0}$ such that $f^{\prime} \in L[a, b]$, where $a, b \in \mathbb{I}$ with $a<b$ and $[a, b] \subset[0, b]$. If $\left|f^{\prime}\right|^{q} \in K_{s}^{2}[a, b]$, for some fixed $s \in(0,1]$ and $q>1$ with $1 / p+1 / q=1$, then for $h \in(0,1)$ with $1 / n \leq h \leq(n-1) / n$ for any $n \geq 2$ the following inequality holds:

$$
\begin{aligned}
\left|S_{a}^{b}(f)(h, n)\right| \leq & (b-a)\left\{\frac{1+(h n-1)^{p+1}}{n^{p+1}(p+1)}\right\}^{1 / p}\left(\frac{h}{s+1}\right)^{1 / q} \\
& \times\left\{\left|f^{\prime}(h b+(1-h) a)\right|^{q}+\left|f^{\prime}(a)\right|^{q}\right\}^{1 / q} \\
& +(b-a)\left\{\frac{1+(n-h n-1)^{p+1}}{n^{p+1}(p+1)}\right\}^{1 / p}\left(\frac{1-h}{s+1}\right)^{1 / q} \\
& \times\left\{\left|f^{\prime}(b)\right|^{q}+\left|f^{\prime}(h b+(1-h) a)\right|^{q}\right\}^{1 / q} .
\end{aligned}
$$

Proof. From Lemma 2.1, using the Hölder inequality we get

$$
\begin{aligned}
\left|S_{a}^{b}(f)(h, n)\right| \leq & (b-a)\left(\int_{0}^{h}\left|t-\frac{1}{n}\right|^{p} d t\right)^{1 / p}\left(\int_{0}^{h}\left|f^{\prime}(t b+(1-t) a)\right|^{q} d t\right)^{1 / q} \\
& +(b-a)\left(\int_{h}^{1}\left|t-\frac{n-1}{n}\right|^{p} d t\right)^{1 / p}\left(\int_{h}^{1}|f(t b+(1-t) a)|^{q} d t\right)^{1 / q} .
\end{aligned}
$$

Since $\left|f^{\prime}\right|^{q} \in K_{s}^{2}([a, b])$ for a fixed $s \in(0,1]$, we have

(a)

$$
\int_{0}^{h}\left|f^{\prime}(t b+(1-t) a)\right|^{q} d t \leq\left(\frac{h}{s+1}\right)\left\{\left|f^{\prime}(h b+(1-h) a)\right|^{q}+\left|f^{\prime}(a)\right|^{q}\right\},
$$


(b)

$$
\int_{h}^{1}\left|f^{\prime}(t b+(1-t) a)\right|^{q} d t \leq\left(\frac{1-h}{s+1}\right)\left\{\left|f^{\prime}(b)\right|^{q}+\left|f^{\prime}(h b+(1-h) a)\right|^{q}\right\} .
$$

By (2.11) and (2.12), the assertion (2.10) holds.

Corollary 2.5. In Theorem 2.4, letting $n=6$ and $h=1 / 2$, one has

$$
\begin{aligned}
\left|S_{a}^{b}(f)\left(\frac{1}{2}, 6\right)\right| \leq & (b-a)\left(\frac{2^{p+1}+1}{6^{p+1}(p+1)}\right)^{1 / p}\left(\frac{1}{2(s+1)}\right)^{1 / q} \\
& \times\left[\left\{\left|f^{\prime}(a)\right|^{q}+\left|f^{\prime}\left(\frac{a+b}{2}\right)\right|^{q}\right\}^{1 / q}+\left\{\left|f^{\prime}\left(\frac{a+b}{2}\right)\right|^{q}+\left|f^{\prime}(b)\right|^{q}\right\}^{1 / q}\right] .
\end{aligned}
$$

Theorem 2.6. Let $f:[0, b] \rightarrow \mathbb{R}, b>0$ be a differentiable mapping on $\mathbb{I}^{0}$ such that $f^{\prime} \in L[a, b]$, where $a, b \in \mathbb{I}$ with $a<b$ and $[a, b] \subset[0, b]$. If $\left|f^{\prime}\right|^{q} \in K_{s}^{2}([a, b])$, for some fixed $s \in(0,1]$ and $q>1$ with $1 / p+1 / q=1$, then for $h \in(0,1)$ with $1 / n \leq h \leq(n-1) / n$ for any $n \geq 2$ the following inequality holds:

$$
\begin{aligned}
\left|S_{a}^{b}(f)(h, n)\right| \leq & (b-a)\left\{\frac{1}{n^{p+1}(p+1)}\right\}^{1 / p}\left(\frac{1}{s+1}\right)^{1 / q} \\
& \times\left[\{ 1 + ( h n - 1 ) ^ { p + 1 } \} ^ { 1 / p } \left\{\left(h^{s+1}\left|f^{\prime}(b)\right|^{q}+\left(1-(1-h)^{s+1}\right)\left|f^{\prime}(a)\right|^{q}\right\}^{1 / q}\right.\right. \\
& +\left\{1+(n-n h-1)^{p+1}\right\}^{1 / p} \times\left\{\left(\left(1-h^{s+1}\right)\left|f^{\prime}(b)\right|^{q}+(1-h)^{s+1}\left|f^{\prime}(a)\right|^{q}\right\}^{1 / q}\right] .
\end{aligned}
$$

Proof. Note that
(a) $\int_{0}^{h}\left|f^{\prime}(t b+(1-t) a)\right|^{q} d t \leq\left(\frac{h^{s+1}}{s+1}\right)\left|f^{\prime}(b)\right|^{q}+\left(\frac{1-(1-h)^{s+1}}{s+1}\right)\left|f^{\prime}(a)\right|^{q}$,
(b) $\int_{h}^{1}\left|f^{\prime}(t b+(1-t) a)\right|^{q} d t \leq\left(\frac{1-h^{s+1}}{s+1}\right)\left|f^{\prime}(b)\right|^{q}+\left(\frac{(1-h)^{s+1}}{s+1}\right)\left|f^{\prime}(a)\right|^{q}$.

By (2.11) and (2.16), the assertion (2.15) of this theorem holds. 
Corollary 2.7. In Theorem 2.6, letting $h=1 / 2$, then one has

$$
\begin{aligned}
\left|S_{a}^{b}(f)\left(\frac{1}{2}, n\right)\right| \leq & (b-a)\left\{\frac{2^{p+1}+(n-2)^{p+1}}{2^{p+1} n^{p+1}(p+1)}\right\}^{1 / p}\left(\frac{1}{2^{s+1}(s+1)}\right)^{1 / q} \\
& \times\left[\left\{\left|f^{\prime}(b)\right|^{q}+\left(2^{s+1}-1\right)\left|f^{\prime}(a)\right|^{q}\right\}^{1 / q}+\left\{\left(2^{s+1}-1\right)\left|f^{\prime}(b)\right|^{q}+\left|f^{\prime}(a)\right|^{q}\right\}^{1 / q}\right],
\end{aligned}
$$

which implies that Theorem 2.6 is a generalization of Theorem 1.1 .

Theorem 2.8. Let $f:[0, b] \rightarrow \mathbb{R}, b>0$ be a differentiable mapping on $\mathbb{I}^{0}$ such that $f^{\prime} \in L[a, b]$, where $a, b \in \mathbb{I}$ with $a<b$ and $[a, b] \subset[0, b]$. If $\left|f^{\prime}\right|^{q} \in K_{s}^{2}([a, b])$, for some fixed $s \in(0,1]$ and $q \geq 1$ with $1 / p+1 / q=1$, then for $h \in(0,1)$ with $1 / n \leq h \leq(n-1) / n$ for any $n \geq 2$ the following inequality holds:

$$
\begin{aligned}
\left|S_{a}^{b}(f)(h, n)\right| \leq & (b-a)\left\{\frac{1+(n h-1)^{2}}{2 n^{2}}\right\}^{1 / p}\left\{\lambda_{21}\left|f^{\prime}(b)\right|^{q}+v_{21}\left|f^{\prime}(a)\right|^{q}\right\}^{1 / q} \\
& +(b-a)\left\{\frac{1+(n h-n+1)^{2}}{2 n^{2}}\right\}^{1 / p}\left\{\lambda_{22}\left|f^{\prime}(b)\right|^{q}+v_{22}\left|f^{\prime}(a)\right|^{q}\right\}^{1 / q}
\end{aligned}
$$

where

$$
\begin{gathered}
\lambda_{21}=\frac{2}{n^{s+2}(s+1)(s+2)}+\frac{h^{s+1}(-2-s+h n+h n s)}{n(s+1)(s+2)}, \\
v_{21}=\frac{2(n-1)^{s+2}}{n^{s+2}(s+1)(s+2)}+\frac{(s+2-n)\left(1+(1-h)^{s+1}\right)}{n(s+1)(s+2)}-\frac{h(1-h)^{s+1}}{s+2}, \\
\lambda_{22}=\frac{2(n-1)^{s+2}}{n^{s+2}(s+1)(s+2)}+\frac{(s+2-n)\left(1+h^{s+1}\right)}{n(s+1)(s+2)}-\frac{h^{s+1}(1-h)}{s+2}, \\
v_{22}=\frac{2}{n^{s+2}(s+1)(s+2)}+\frac{(1-h)^{s+1}(-2-s+n-h n+n s-h n s)}{n(s+1)(s+2)} .
\end{gathered}
$$

Proof. Suppose that $q \geq 1$. From Lemma 2.1, using the power mean inequality one has

$$
\begin{aligned}
\left|S_{a}^{b}(f)(h, n)\right| \leq(b-a) & {\left[\left(\int_{0}^{h}\left|t-\frac{1}{n}\right| d t\right)^{1 / p}\left(\int_{0}^{h}\left|t-\frac{1}{n}\right|\left|f^{\prime}(t b+(1-t) a)\right|^{q} d t\right)^{1 / q}\right.} \\
& \left.+\left(\int_{h}^{1}\left|t-\frac{n-1}{n}\right| d t\right)^{1 / p}\left(\int_{h}^{1}\left|t-\frac{n-1}{n}\right|\left|f^{\prime}(t b+(1-t) a)\right|^{q} d t\right)^{1 / q}\right] .
\end{aligned}
$$


Since $\left|f^{\prime}\right|$ is s-convex on $[a, b]$, we have

$$
\begin{gathered}
\int_{0}^{h}\left|t-\frac{1}{n}\right|\left|f^{\prime}(t b+(1-t) a)\right|^{q} d t \leq \lambda_{21}\left|f^{\prime}(b)\right|^{q}+v_{21}\left|f^{\prime}(a)\right|^{q} \\
\int_{h}^{1}\left|t-\frac{n-1}{n}\right||f(t b+(1-t) a)|^{q} d t \leq \lambda_{22}\left|f^{\prime}(b)\right|^{q}+v_{22}\left|f^{\prime}(a)\right|^{q} .
\end{gathered}
$$

By the above facts (2.20) and (2.21), the assertion (2.18) in this theorem is proved.

Corollary 2.9. In Theorem 2.8 , letting $h=1 / 2$, one has

$$
\begin{aligned}
& \lambda_{21}=v_{22}=\frac{2}{n^{s+2}(s+1)(s+2)}+\frac{(n-2)(s+1)-2}{2^{s+2} n(s+1)(s+2)} \\
& \lambda_{22}=v_{21}=\frac{n^{s+2} 2^{-(s+2)}(s+1)+2(n-1)^{s+2}}{n^{s+2}(s+1)(s+2)}+\frac{(s-n+2)\left(2^{s+1}+1\right)}{2^{s+1} n(s+1)(s+2)},
\end{aligned}
$$

which implies that

$$
\begin{aligned}
\left|S_{a}^{b}(f)\left(\frac{1}{2}, n\right)\right| \leq & (b-a)\left(\frac{1}{8}-\frac{1}{2 n}+\frac{1}{n^{2}}\right)^{1 / p} \\
& \times\left[\left\{\lambda_{21}\left|f^{\prime}(b)\right|^{q}+\lambda_{22}\left|f^{\prime}(a)\right|^{q}\right\}^{1 / q}+\left\{\lambda_{22}\left|f^{\prime}(b)\right|^{q}+\lambda_{21}\left|f^{\prime}(a)\right|^{q}\right\}^{1 / q}\right] .
\end{aligned}
$$

Especially, in Theorem 2.8, letting $h=1 / 2$ and $m=1$, one has

$$
\begin{aligned}
\left|S_{a}^{b}(f)\left(\frac{1}{2}, n\right)\right| \leq & (b-a)\left(\frac{1}{8}-\frac{1}{2 n}+\frac{1}{n^{2}}\right)^{1 / p} \\
& \times\left\{\left(\lambda_{21}\left|f^{\prime}(b)\right|^{q}+\lambda_{22}\left|f^{\prime}(a)\right|^{q}\right)^{1 / q}\right. \\
& \left.\quad+\left(\lambda_{22}\left|f^{\prime}(b)\right|^{q}+\lambda_{21}\left|f^{\prime}(a)\right|^{q}\right)^{1 / q}\right\} .
\end{aligned}
$$

\section{Applications to Special Means}

We now consider the applications of our theorems to the followings special means.

(a) The arithmetic mean: $A(a, b)=(a+b) / 2, a, b \geq 0$.

(b) The $p$-logarithmic mean:

$$
L_{p}(a, b)= \begin{cases}{\left[\frac{b^{p+1}-a^{p+1}}{(p+1)(b-a)}\right]^{1 / p},} & \text { if } a \neq b, \\ a, & \text { if } a=b\end{cases}
$$


for $p \in \mathbb{R} \backslash\{-1,0\}$ and $a, b>0$.

Now, using the results of Section 2, some new inequalities are derived for the following means:

(1.1) Let $f:[a, b] \rightarrow \mathbb{R},(0<a<b), f(x)=x^{s}, s \in(0,1]$.

(a) In Theorem 2.2,

(i) if $h=1 / 2$ and $n \geq 2$, then we get

$$
\begin{aligned}
& \left|\frac{1}{n}\left[2 A\left(a^{s}, b^{s}\right)+(n-2) A^{s}(a, b)\right]-L_{s}^{s}(a, b)\right| \\
& \quad \leq(b-a) 2 s\left\{\frac{2+2(n-1)^{s+2}+n^{s+1}\left(2+s-n-2^{-(s+1)} n\right)}{n^{s+2}(s+1)(s+2)}\right\} A\left(a^{s-1}, b^{s-1}\right),
\end{aligned}
$$

and,

(ii) if $h=1 / 2$ and $n=6$, then we have

$$
\left|\frac{1}{3}\left[A\left(a^{s}, b^{s}\right)+2 A^{s}(a, b)\right]-L_{s}^{s}(a, b)\right| \leq(b-a) s\left\{\frac{6^{-s}+5^{s+2} 6^{-s}+3\left(s-4-2^{-s} 3\right)}{9(s+1)(s+2)}\right\} A\left(a^{s-1}, b^{s-1}\right) .
$$

(b) In Theorem 2.4,

(i) if $h=1 / 2, n \geq 2$ and $q>1$ then we get:

$$
\begin{aligned}
\mid \frac{1}{n}[2 A & \left.\left(a^{s}, b^{s}\right)+(n-2) A^{s}(a, b)\right]-L_{s}^{s}(a, b) \mid \\
\leq & \frac{b-a}{2}\left\{\frac{1+((n / 2)-1)^{p+1}}{n^{p+1}(p+1)}\right\}^{1 / p}\left(\frac{s^{q}}{2(s+1)}\right)^{1 / q} \\
& \left.\left.\times\left[\left\{A^{(s-1) q}(A(a, b))+a^{(s-1) q}\right)\right\}^{1 / q}+\left\{A^{(s-1) q}(A(a, b))+b^{(s-1) q}\right)\right\}^{1 / q}\right],
\end{aligned}
$$

and

(ii) if $h=1 / 2, n=6$ and $q>1$, then we have

$$
\begin{aligned}
\left|\frac{1}{3}\left[A\left(a^{s}, b^{s}\right)+2 A^{s}(a, b)\right]-L_{s}^{s}(a, b)\right| \\
\leq \frac{b-a}{12}\left\{\frac{1+2^{p+1}}{3(p+1)}\right\}^{1 / p}\left(\frac{s^{q}}{(s+1)}\right)^{1 / q} \\
\left.\left.\quad \times\left[\left\{A^{(s-1) q}(A(a, b))+a^{(s-1) q}\right)\right\}^{1 / q}+\left\{A^{(s-1) q}(A(a, b))+b^{(s-1) q}\right)\right\}^{1 / q}\right],
\end{aligned}
$$

(c) In Theorem 2.6, 
International Journal of Mathematics and Mathematical Sciences

(i) if $h=1 / 2, n \geq 2$ and $q>1$ then we get

$$
\begin{aligned}
\left|\frac{1}{n}\left[2 A\left(a^{s}, b^{s}\right)+(n-2) A^{s}(a, b)\right]-L_{s}^{s}(a, b)\right| \\
\leq(b-a)\left\{\frac{1+((n / 2)-1)^{p+1}}{n^{p+1}(p+1)}\right\}^{1 / p}\left\{\frac{s^{q}}{2^{s+1}(s+1)}\right\}^{1 / q} \\
\quad \times\left[\left\{b^{(s-1) q}+\left(2^{s+1}-1\right) a^{(s-1) q}\right\}^{1 / q}+\left\{\left(2^{s+1}-1\right) b^{(s-1) q}+a^{(s-1) q}\right\}^{1 / q}\right],
\end{aligned}
$$

and

(ii) if $h=1 / 2, n=6$ and $q>1$, then we have

$$
\begin{aligned}
& \left|\frac{1}{3}\left[A\left(a^{s}, b^{s}\right)+2 A^{s}(a, b)\right]-L_{s}^{s}(a, b)\right| \\
& \leq(b-a)\left\{\frac{1+2^{p+1}}{6^{p+1}(p+1)}\right\}^{1 / p}\left\{\frac{s^{q}}{2^{s+1}(s+1)}\right\}^{1 / q} \\
& \quad \times\left[\left\{b^{(s-1) q}+\left(2^{s+1}-1\right) a^{(s-1) q}\right\}^{1 / q}+\left\{\left(2^{s+1}-1\right) b^{(s-1) q}+a^{(s-1) q}\right\}^{1 / q}\right] .
\end{aligned}
$$

In Theorem 2.8,

(i) if $h=1 / 2, n \geq 2$ and $q \geq 1$ then we get

$$
\begin{aligned}
\left|\frac{1}{n}\left[2 A\left(a^{s}, b^{s}\right)+(n-2) A^{s}(a, b)\right]-L_{S}^{s}(a, b)\right| \\
\leq(b-a)\left\{\frac{1+((n / 2)-1)^{2}}{2 n^{2}}\right\}^{1 / p} \\
\quad \times\left[\left\{\lambda_{21}^{\prime} b^{(s-1) q}+\lambda_{22}^{\prime} a^{(s-1) q}\right\}^{1 / q}+\left\{\lambda_{22}^{\prime} b^{(s-1) q}+\lambda_{21}^{\prime} b^{(s-1) q}\right\}^{1 / q}\right],
\end{aligned}
$$

where

$$
\begin{aligned}
& \lambda_{21}^{\prime}=\frac{2}{n^{s+2}(s+1)(s+2)}+\frac{(n s / 2)+(n / 2)-s-2}{n 2^{s+1}(s+1)(s+2)}, \\
& \lambda_{22}^{\prime}=\frac{2(n-1)^{s+2}}{n^{s+2}(s+1)(s+2)}+\frac{(s-n+2)\left(2^{s+1}+1\right)}{n 2^{s+1}(s+1)(s+2)}-\frac{1}{2^{s+2}(s+2)},
\end{aligned}
$$


and

(ii) if $h=1 / 2, n=6$ and $q \geq 1$, then we have

$$
\begin{aligned}
& \left|\frac{1}{3}\left\{A\left(a^{s}, b^{s}\right)+2 A^{s}(a, b)\right\}-L_{s}^{s}(a, b)\right| \\
& \quad \leq(b-a)\left(\frac{5}{72}\right)^{1 / p}\left[\left\{\lambda_{21}^{\prime \prime} b^{(s-1) q}+\lambda_{22}^{\prime \prime} a^{(s-1) q}\right\}^{1 / q}+\left\{\lambda_{22}^{\prime \prime} b^{(s-1) q}+\lambda_{21}^{\prime \prime} b^{(s-1) q}\right\}^{1 / q}\right],
\end{aligned}
$$

where

$$
\begin{aligned}
& \lambda_{21}^{\prime \prime}=\frac{2+3^{s+1}(2 s+1)}{6^{s+2}(s+1)(s+2)} \\
& \lambda_{22}^{\prime \prime}=\frac{2 \cdot 5^{s+2}+3^{s+1}\left(2^{s+1}-2\right) s-3^{s+1}\left(2^{s+3}+7\right)}{6^{s+2}(s+1)(s+2)} .
\end{aligned}
$$

(2.2) Let $f:[a, b] \rightarrow \mathbb{R},(0<a<b), f(x)=1 / x^{s}, s \in(0,1]$.

(a) In Theorem 2.2,

(i) if $h=1 / 2$ and $n \geq 2$, then we get

$$
\begin{aligned}
\left|\frac{1}{n}\left[2 A\left(a^{-s}, b^{-s}\right)+(n-2) A^{-s}(a, b)\right]-L_{-s}^{-s}(a, b)\right| \\
\leq(b-a) 2 s\left\{\frac{2^{s+2}\left(1+(n-1)^{s+2}\right)+2^{s+1} n^{s+1}(2+s-n)-n^{s+2}}{n^{s+2} 2^{s+1}(s+1)(s+2)}\right\} \\
\quad \times A\left(a^{-(s+1)}, b^{-(s+1)}\right),
\end{aligned}
$$

and

(ii) if $h=1 / 2$ and $n=6$, then we have

$$
\begin{aligned}
& \left|\frac{1}{3}\left[A\left(a^{-s}, b^{-s}\right)+2 A^{-s}(a, b)\right]-L_{-s}^{-s}(a, b)\right| \\
& \quad \leq \frac{2}{3}(b-a) s\left\{\frac{1+5^{s+2}+3(s-4) 6^{s}-3^{s+2}}{6^{s+2}(s+1)(s+2)}\right\} A\left(a^{-(s+1)}, b^{-(s+1)}\right) .
\end{aligned}
$$

(b) In Theorem 2.4, 
International Journal of Mathematics and Mathematical Sciences

(i) if $h=1 / 2$ and $n \geq 2$, then we get

$$
\begin{aligned}
\mid \frac{1}{n}[2 & \left.A\left(a^{-s}, b^{-s}\right)+(n-2) A^{-s}(a, b)\right]-L_{-s}^{-s}(a, b) \mid \\
\leq & (b-a)\left\{\frac{1+((n / 2)-1)^{p+1}}{n^{p+1}(p+1)}\right\}^{1 / p}\left\{\frac{s^{q}}{2(s+1)}\right\}^{1 / q} \\
& \times\left[\left\{A^{-(s+1) q}(a, b)+a^{-(s+1) q}\right\}^{1 / q}+\left\{A^{-(s+1) q}(a, b)+b^{-(s+1) q}\right\}^{1 / q}\right],
\end{aligned}
$$

and

(ii) if $h=1 / 2$ and $n=6$, then we have

$$
\begin{aligned}
\left|\frac{1}{3}\left[A\left(a^{-s}, b^{-s}\right)+2 A^{-s}(a, b)\right]-L_{-s}^{-s}(a, b)\right| \\
\leq(b-a)\left\{\frac{1+2^{p+1}}{6^{p+1}(p+1)}\right\}^{1 / p}\left\{\frac{s^{q}}{(s+1)}\right\}^{1 / q} \\
\quad \times\left\{A^{1 / q}\left(A^{-(s+1) q}(a, b), a^{-(s+1) q}\right)+A^{1 / q}\left(A^{-(s+1) q}(a, b), b^{-(s+1) q}\right)\right\} .
\end{aligned}
$$

(c) In Theorem 2.6,

(i) if $h=1 / 2$ and $n \geq 2$, then we get

$$
\begin{aligned}
\left|\frac{1}{n}\left[2 A\left(a^{-s}, b^{-s}\right)+(n-2) A^{-s}(a, b)\right]-L_{-s}^{-s}(a, b)\right| \\
\leq(b-a)\left\{\frac{1}{n^{p+1}(p+1)}\right\}^{1 / p}\left\{\frac{s^{q}}{2^{s+1}(s+1)}\right\}^{1 / q}\left\{1+\left(\frac{n}{2}-1\right)^{p+1}\right\}^{1 / p} \\
\quad \times\left[\left\{b^{-(s+1) q}+\left(2^{s+1}-1\right) a^{-(s+1) q}\right\}^{1 / q}+\left\{\left(2^{s+1}-1\right) b^{-(s+1) q}+a^{-(s+1) q}\right\}^{1 / q}\right],
\end{aligned}
$$

and

(ii) if $h=1 / 2$ and $n=6$, then we have

$$
\begin{aligned}
& \left|\frac{1}{3}\left[A\left(a^{-s}, b^{-s}\right)+2 A^{-s}(a, b)\right]-L_{-s}^{-s}(a, b)\right| \\
& \leq(b-a)\left\{\frac{1+2^{p+1}}{6^{p+1}(p+1)}\right\}^{1 / p}\left\{\frac{s^{q}}{2^{s+1}(s+1)}\right\}^{1 / q} \\
& \quad \times\left[\left\{b^{-(s+1) q}+\left(2^{s+1}-1\right) a^{-(s+1) q}\right\}^{1 / q}+\left\{\left(2^{s+1}-1\right) b^{-(s+1) q}+a^{-(s+1) q}\right\}^{1 / q}\right] .
\end{aligned}
$$


(d) In Theorem 2.8,

(i) if $h=1 / 2, n \geq 2$ and $q \geq 1$ then we get

$$
\begin{aligned}
\mid \frac{1}{n}[2 A & \left.\left(a^{-s}, b^{-s}\right)+2^{-s}(n-2) A^{-s}(h b,(1-h) a)\right]-L_{-s}^{-s}(a, b) \mid \\
\leq & (b-a) s\left\{\frac{(n-2)^{2}+4}{8 n^{2}}\right\}^{1 / p} \\
& \times\left[\left\{\lambda_{31}^{\prime} b^{(s-1) q}+\lambda_{32}^{\prime} a^{(s-1) q}\right\}^{1 / q}+\left\{\lambda_{32}^{\prime} b^{(s-1) q}+\lambda_{31}^{\prime} b^{(s-1) q}\right\}^{1 / q}\right],
\end{aligned}
$$

where

$$
\begin{aligned}
& \lambda_{31}^{\prime}=\frac{2}{n^{s+2}(s+1)(s+2)}+\frac{-2-s+n / 2+n s / 2}{n 2^{s+2}(s+1)(s+2)} \\
& \lambda_{32}^{\prime}=\frac{2(n-1)^{s+2}}{n^{s+2}(s+1)(s+2)}+\frac{(s-n+2)\left(2^{s+1}+1\right)}{n 2^{s+1}(s+1)(s+2)}-\frac{1}{2^{s+2}(s+2)}
\end{aligned}
$$

and

(ii) if $h=1 / 2, n=6$ and $q \geq 1$, then we have

$$
\begin{aligned}
& \left|\frac{1}{3}\left[A\left(a^{-s}, b^{-s}\right)+2 A^{-s}(a, b)\right]-L_{-s}^{-s}(a, b)\right| \\
& \quad \leq(b-a) s\left\{\frac{5}{72}\right\}^{1 / p} \times\left[\left\{\lambda_{31}^{\prime \prime} b^{q(s-1)}+\lambda_{32}^{\prime \prime} a^{q(s-1)}\right\}^{1 / q}+\left\{\lambda_{32}^{\prime \prime} b^{q(s-1)}+\lambda_{31}^{\prime \prime} b^{q(s-1)}\right\}^{1 / q}\right],
\end{aligned}
$$

where

$$
\begin{aligned}
& \lambda_{31}^{\prime \prime}=\frac{12+3^{s+2}(2 s+1)}{6^{s+3}(s+1)(s+2)} \\
& \lambda_{32}^{\prime \prime}=\frac{2 \cdot 5^{s+2}+3^{s+1}\left(2^{s+1}-4\right) s+3^{s+1}\left(1-2^{s+3}\right)}{6^{s+2}(s+1)(s+2)} .
\end{aligned}
$$

\section{Acknowledgments}

The author is thankful to Professor Merve Avci and Uğur S. Kirmaci, Atatürk University, K. K. Education Faculty, Deptartment of Mathematics, for their valuable suggestions and for the improvement of this paper. This work is financially supported by Hanseo University research fund no. 111-S-101. 


\section{References}

[1] J. Park, "Inequalities for some differentiable convex mappings," Far East Journal of Mathematical Sciences, vol. 44, no. 2, pp. 251-259, 2010.

[2] J. Park, "Ostrowski type inequalities for mappings whose derivatives are $(s, m)$-convex in the second sense," Far East Journal of Mathematical Sciences, vol. 49, no. 2, pp. 181-195, 2011.

[3] J. Park, "Generalization of Ostrowski-type inequalities for differentiable real $(s, m)$-convex mappings," Far East Journal of Mathematical Sciences, vol. 49, no. 2, pp. 157-171, 2010.

[4] M. Z. Sarikaya, E. Set, and M. E. Özdemir, “On new inequalities of Simpson's type for s-convex functions," Computers and Mathematics with Applications, vol. 60, no. 8, pp. 2191-2199, 2010.

[5] E. Set, M. E. Özdemir, and M. Z. Sarikaya, "Inequalities of Hermite-hadamard's type for functions whose derivatives absolute values are m-convex," AIP Conference Proceedings, vol. 1309, no. 1, pp. 861873, 2010.

[6] J. Park, "Generalizations of some Simpson-like type inequalities via differentiable s-convex mappings in the second sense," Far East Journal of Mathematical Sciences, vol. 54, no. 2, pp. 201-215, 2011.

[7] U. S. Kirmaci, M. K. Bakula, M. E. Özdemir, and J. Pečarić, "Hadamard-type inequalities for s-convex functions," Applied Mathematics and Computation, vol. 193, no. 1, pp. 26-35, 2007.

[8] M. Alomari, M. Darus, and S. S. Dragomir, "New inequalities of Simpson's type for s-convex functions with applications," Research Group in Mathematical Inequalities and Applications, vol. 12, no. 4, 2009.

[9] S. S. Dragomir, R. P. Agarwal, and P. Cerone, "On Simpson's inequality and applications," Journal of Inequalities and Applications, vol. 5, no. 6, pp. 533-579, 2000. 


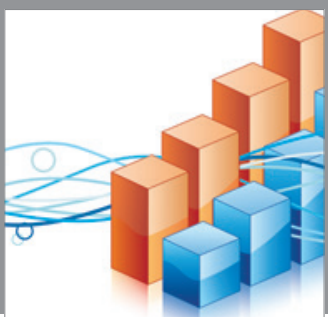

Advances in

Operations Research

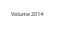

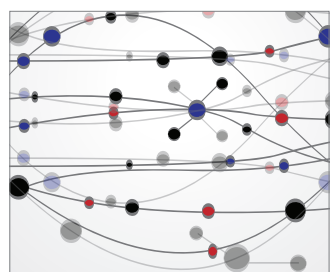

\section{The Scientific} World Journal
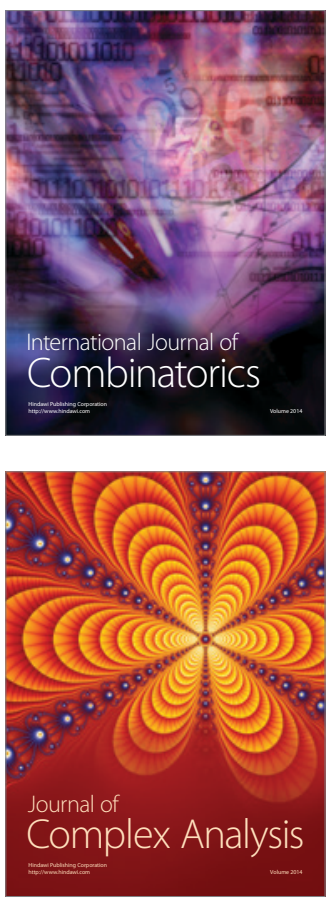

International Journal of

Mathematics and

Mathematical

Sciences
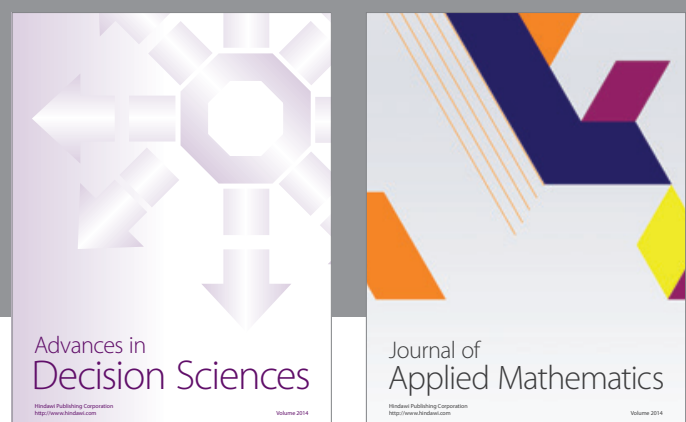

Journal of

Applied Mathematics
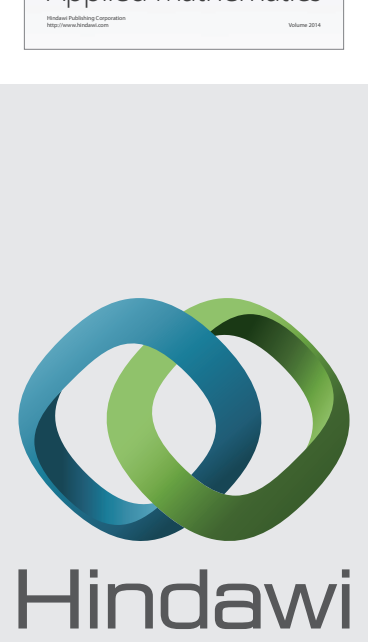

Submit your manuscripts at http://www.hindawi.com
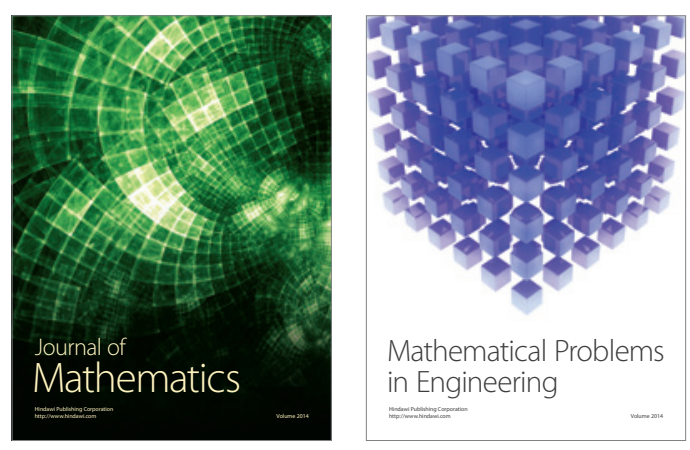

Mathematical Problems in Engineering
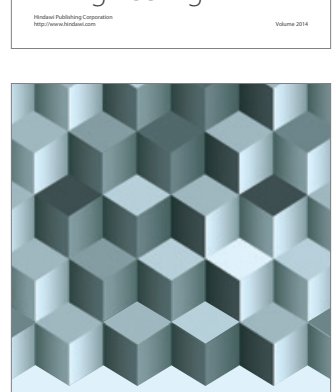

Journal of

Function Spaces
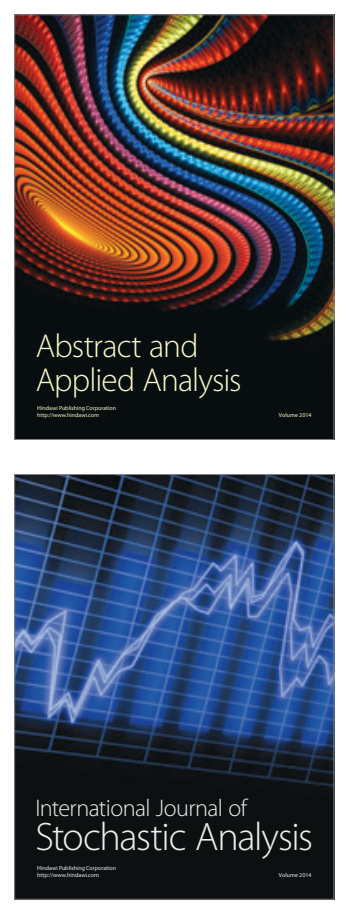

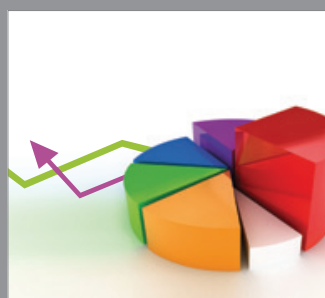

ournal of

Probability and Statistics

Promensencen
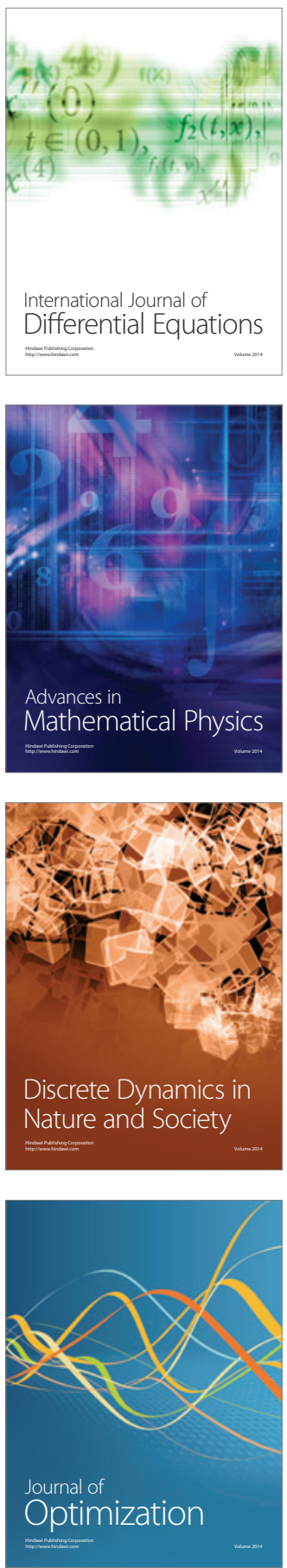